\title{
Augmented Reality in Esthetic Dentistry: a Case Report
}

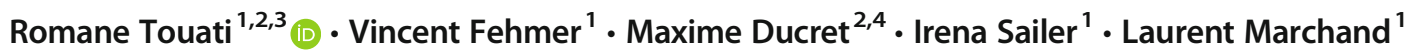

Accepted: 23 March 2021 / Published online: 26 April 2021

(C) The Author(s) 2021

\begin{abstract}
Purpose of Review The aim of this case report was to illustrate the clinical procedure integrating augmented reality (AR) for complex patient cases requiring full mouth rehabilitation.

Recent Findings The introduction of AR technology to the fields of medicine and dentistry has led to numerous applications in education, surgery, and esthetics. Recently, a new AR software was introduced in esthetic dentistry which allows for real-time smile projection and thus improves communication with patients and the dental laboratory.

Summary The presented case shows a patient with multiple missing teeth, diastemata, and an impaired masticatory and phonetic ability. After reconstruction of the posterior zone, the AR software was used for the conception of the esthetic zone, integrating the patient into the decision-making process. The result was an esthetic rehabilitation applying palatal and buccal veneers which corresponded to the chosen AR design. The patient appreciated the opportunity to pre-visualize a possible final outcome in an interactive way which increased his confidence in the chosen treatment. Further studies are needed to assess the precision and reproducibility of the described protocol.
\end{abstract}

Keywords Augmented reality $\cdot$ AR $\cdot \mathrm{CAD} / \mathrm{CAM} \cdot$ Virtual $\cdot$ IvoSmile

\section{Introduction}

Augmented reality (AR) is a technology that combines the virtual and physical worlds and therefore augments the realworld experience [1]. Although the term "augmented reality" has been coined in the early 1990s, the origins of the idea to merge the digital and physical worlds date back to 30 years before. Early predecessors to modern AR technology were

This article is part of the Topical Collection on Modern Production Laboratory Advances in Dental Technology

Romane Touati

touati.romane@gmail.com

1 Division of Fixed Prosthodontics and Biomaterials, University Clinic of Dental Medicine, University of Geneva, Geneva, Switzerland

2 Hospices Civils de Lyon, Service de Consultations et Traitements Dentaires, Lyon, France

3 Division de Prothèse Fixe et Biomatériaux, Clinique Universitaire de Médecine Dentaire, Université de Genève, 1, Rue Michel-Servet, 1211 Genève4, Suisse, Switzerland

4 Faculty of Odontology, University Claude Bernard Lyon 1, University of Lyon, Lyon, France originally invented for aerospace engineering [2] and related industrial applications [3]. With the rapid evolution of computer and camera technology around the turn of the millennium however, the scope of application for AR has increased significantly. Apart from industrial uses, AR has since been introduced into the fields of medicine and dentistry with a multitude of emerging applications. Today, AR is used in dentistry for educational purposes [4•], providing a tool to objectively evaluate students and give them direct feedback. Further developments of AR technology have led to its application in oral guided surgery or pre-operative planification $[4 \cdot, 5,6]$.

In esthetic dentistry, the attainment of a patient-specific optimal appearance of the planned restorations may be considered an important objective. A pre-visualization can be achieved by means of a conventional laboratory-made waxup and intraoral mock-up, in the form of a two-dimensional smile design by overlapping idealized teeth forms onto a portrait picture of the patient [7•]. Recently, a procedure using a three-dimensional facial scan of the patient was proposed, but this requires a lot of time and the ability to combine different software, which is not yet simple for all the prosthetic laboratories [8-10]. To overcome this problem, a solution using AR software was proposed, offering fast $3 \mathrm{D}$ conception and promising results $[11,12]$. However, this approach is currently 
limited to the design and conception of teeth in the anterior maxilla. In more complex patient cases, such pre-visualization is often difficult to implement due to a multitude of variables that must be considered. In situations where a full mouth rehabilitation is indicated, there is currently no protocol for the application of AR technology.

The present case report proposes to illustrate the application, as well as the advantages and current drawbacks of AR technology for esthetic full mouth reconstruction.

\section{Case Illustration}

A 59-year-old male patient searched for treatment at the University Clinic for Dental Medicine of Geneva with the primary wish to improve his masticatory and phonetic function as well as the esthetic appearance of his smile. The patient was healthy, took no medication, but was a former smoker with a history of periodontitis. The patient had lost all premolar and molar teeth in the upper arch along with the upper left canine due to periodontitis (Fig. 1). In the lower arch, all molar teeth were missing as well as the right central incisor (tooth 41, Fig. 1). Due to the lack of posterior support, the remaining dentition was heavily worn down with multiple areas of exposed dentine. Furthermore, due to lack of interproximal contacts, multiple visible diastemata opened up impairing the patient's smile.

Apart from the esthetic aspect, the diastemata disturbed the patient's phonetic ability as well as the ability to play brass instruments, which is his favorite passion. As the patient's intraoral situation deteriorated slowly over a period of several decades, the patient became anxious about undergoing a full mouth rehabilitation. The patient had been restored with removable partial dentures in the past, but ultimately never wore them due to lack of comfort. He therefore had high expectations of a possible fixed rehabilitation in terms of esthetic appearance, function, and comfort. At the same time, the patient was having difficulty formulating his opinion on how his teeth should look like as he had been with worn down teeth for decades. The ability to pre-visualize a possible final outcome and discuss the treatment goals was, therefore, of great importance.

For situations where purely an esthetic correction is desired, AR technology has been applied successfully to provide such pre-visualization [12]. As AR software uses existing teeth as fixpoints to provide a predictable smile projection, its use in the present case was limited and inaccurate. For this reason, an initial approach using a conventional wax-up and mock-up was chosen to define the goal of the treatment which served as a guideline for the restorative team (Fig. 2).

The final treatment plan was to first reconstruct the posterior areas in both jaws with implant-supported restorations to stabilize the bite. For the rehabilitation of the maxillary anterior zone, an adhesive approach using palatal and vestibular veneers was planned. This combined sandwich technique allowed for a tissue-preserving preparation of the elongated and periodontally involved maxillary incisors. In the anterior mandible, direct composites were planned to reconstruct the incisal edges, and a resin-bonded bridge with a one wing design was to replace the missing tooth 41 .

The initial mock-up was used as a reference for implant placement (tissue level implants, Straumann, Basel, Switzerland) in the posterior regions. After an uneventful healing period, digitally designed and computer-assisted-
Fig. 1 Initial situation. a Frontal view. b Occlusal view of the upper arch. c Occlusal view of the lower arch
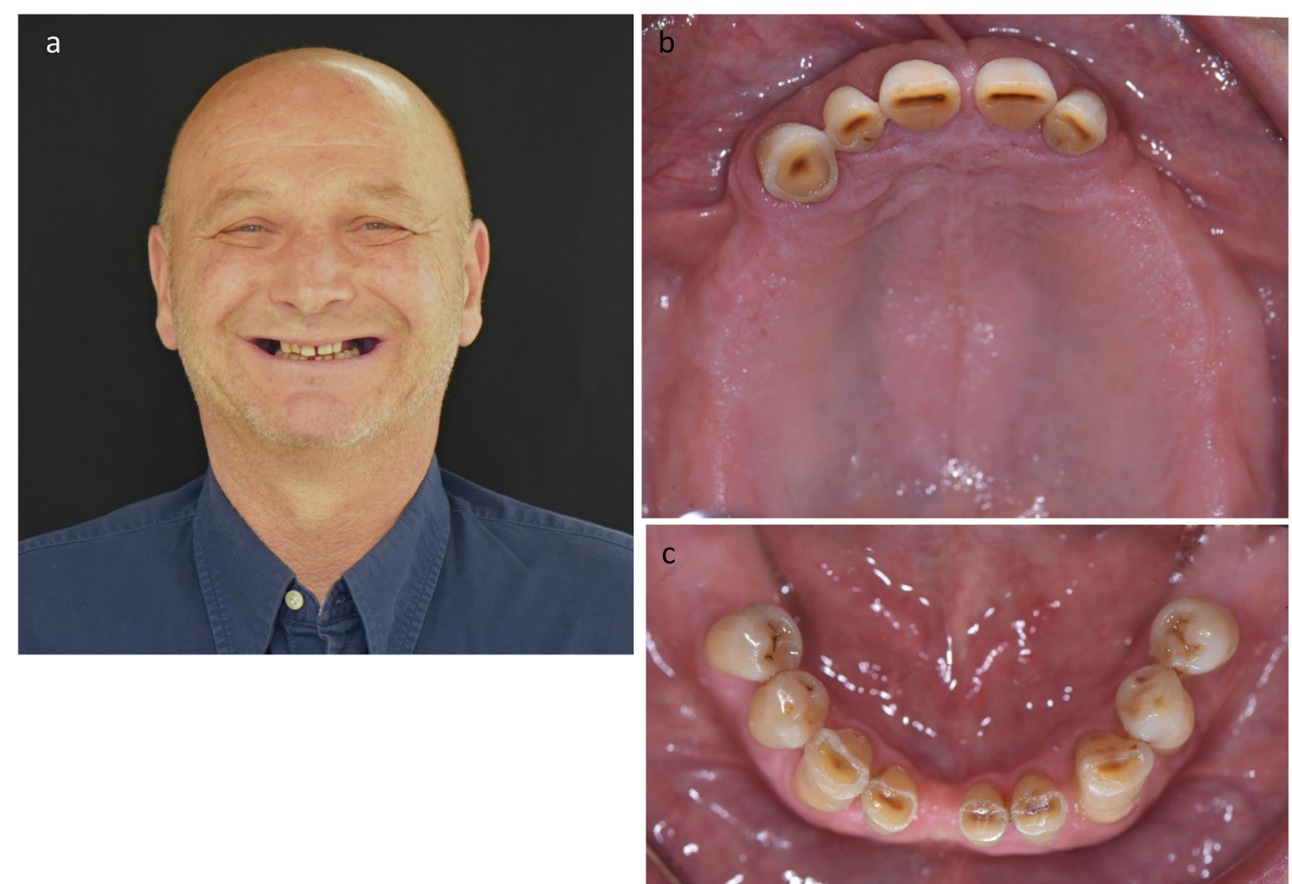
Fig. 2 Conception and reconstruction of posterior teeth. a Set-up of the posterior teeth. b) Intraoral view of the try-in of the set-up. c Frontal view
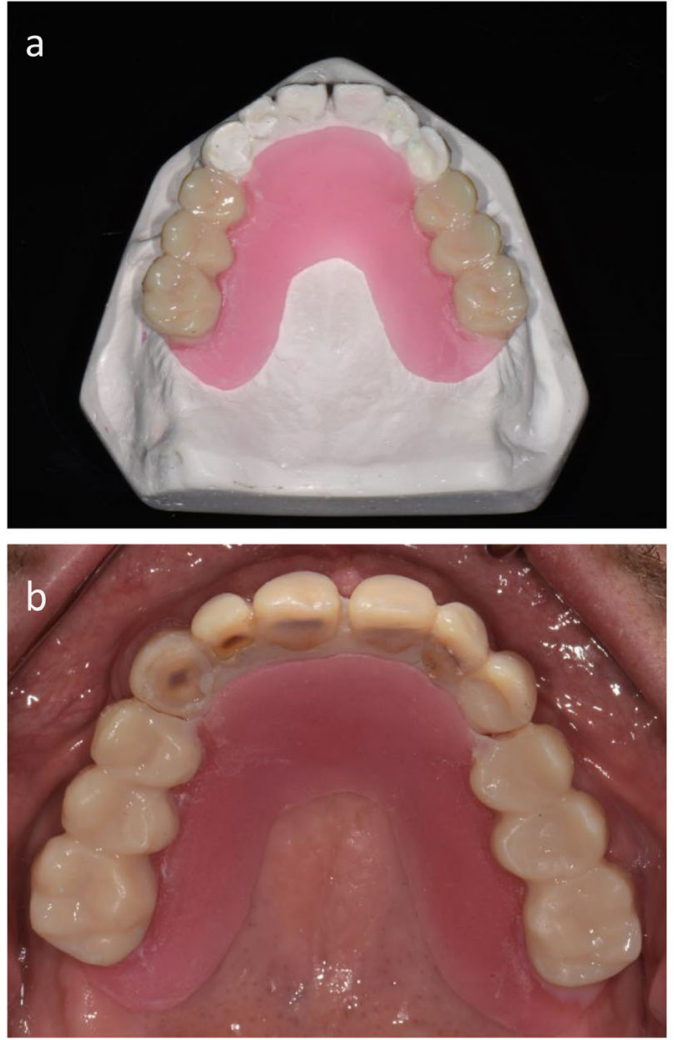

manufactured reconstructions with a cobalt-chromium framework (Coron cobalt-chromium alloy, Straumann, Switzerland) and feldspathic ceramic veneering were fabricated (Fig. 3). This re-established a correct vertical dimension of occlusion (VDO) and stabilized the bite. Before starting the rehabilitation of the esthetic zone however, a smile proposal using an AR software (IvoSmile, Ivoclar Vivadent, Schaan, Liechtenstein) was realized in collaboration with the patient (Fig. 4). This co-diagnostic session represented a discussion platform where the patient could evaluate and visualize his opinion regarding the future position, length, and shape of his teeth. The AR software allowed for projecting different teeth length, width, and color onto the patients face in real time [11]. As the posterior restorations were already in place, the AR projection could be performed accurately, using teeth and restorations as fixpoints. The patient could modify the smile projection until he felt comfortable with the result, and an overall harmonious result was achieved (Fig. 4). The chosen AR projection was saved and later imported into a computer-assisted design (CAD) software and matched with intraoral scan data. Using the multiple 2D pictures including the smile proposal out of the IvoSmile app to create the patient driven design accordingly. A physical model of this was then 3D-printed and used for a physical mock-up to confirm the AR smile projection clinically (Fig. 5). After the patient validated the proposition, a silicone key of the digital wax-up was fabricated to analyze the necessary tooth preparation in all dimensions. To ensure a minimally invasive preparation, only a small amount of tooth substance had to be removed using a diamond bur to respect the minimal material thickness of the final restoration. Furthermore, due to the missing volume in both palatal and buccal dimensions, the final restorations
Fig. 3 Intraoral view of the implant-supported reconstruction of the posterior teeth. a Right. $\mathbf{b}$ Left
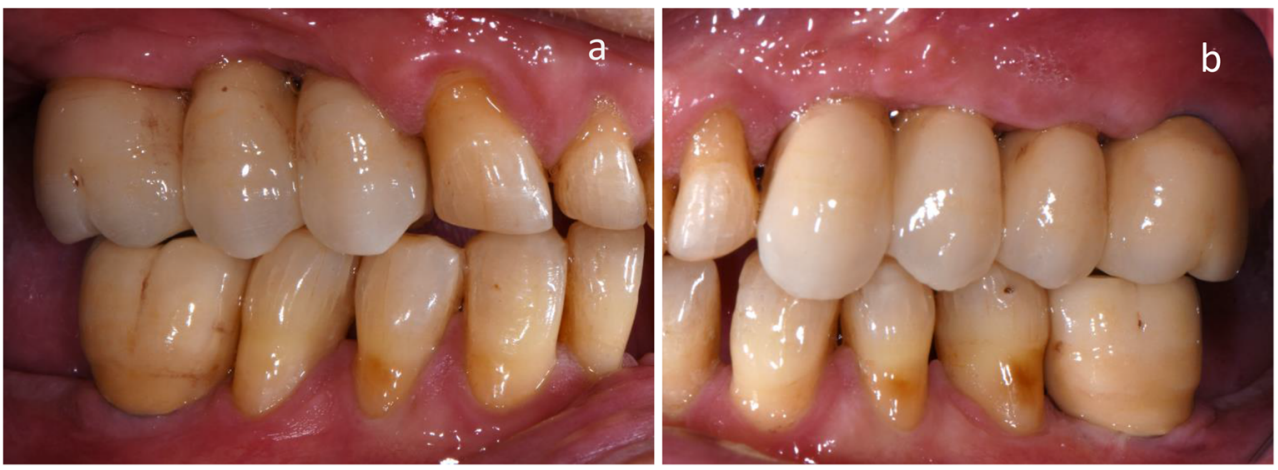


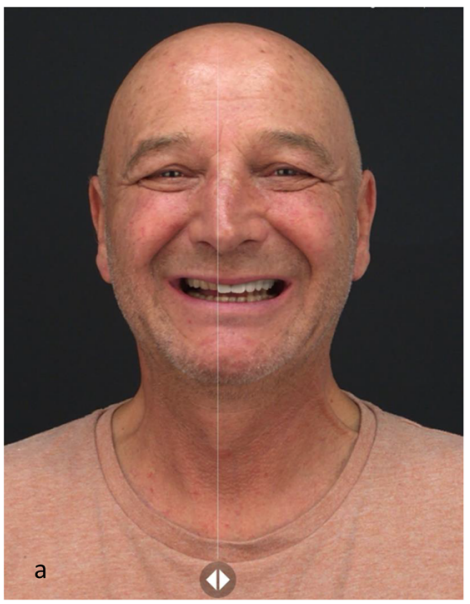

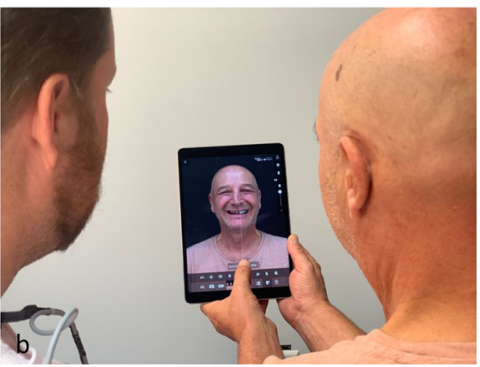

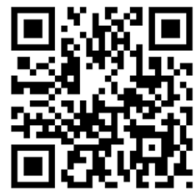

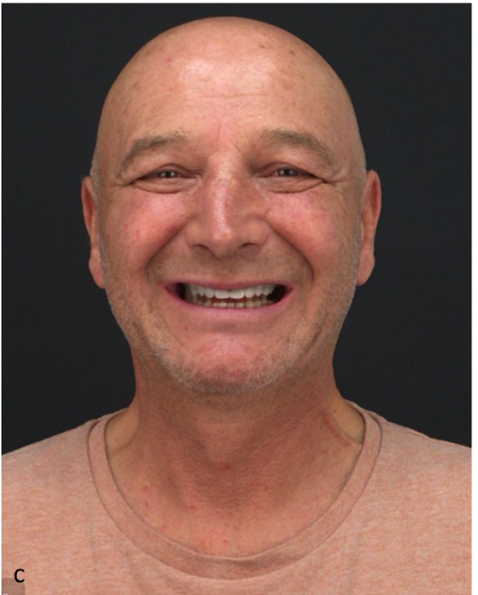

Fig. 4 Co-diagnostic session using an augmented reality protocol. a Real-time superimposition of the virtual mock up. b Discussion platform offered by the augmented reality tool. c Commonly chosen smile projection

could be designed additively, thus requiring very little tooth preparation. The palatal veneers were fabricated out of a polymer-infiltrated ceramic network (PICN) material (Vita Enamic, Vita Zahnfabrik, Bad Säckingen, Germany) and adhesively cemented using pre-heated resin composite (Tetric 210 A2, Ivoclar Vivadent, Liechtenstein). The buccal veneers were then manufactured out of lithium-disilicate (IPS e.max CAD, Ivoclar Vivadent, Schaan, Liechtenstein) and microveneered on the buccal aspect for an optimal esthetic result. Adhesive cementation of the restorations was performed with a dual-curing resin cement (Variolink Esthetic, Ivoclar Vivadent, Liechtenstein). The one wing framework for resin- bonded bridge to replace tooth 41 was fabricated out of zirconia (Lava Plus, 3M ESPE, St. Paul, MN, USA) and buccally veneered with feldspathic ceramic. The finalized bridge was cemented with a self-curing resin cement containing MDP molecules (Panavia 21, Kuraray Noritake Dental Inc., Tokyo, Japan) for optimal adhesion to zirconia [13].

After completion of the treatment, the patient was very happy with the final result (Fig. 6). He appreciated the opportunity to pre-visualize a possible final outcome in such an interactive way during treatment. Furthermore, the potential to influence the decision-making process increased his adherence in the chosen treatment.
Fig. 5 Virtual conception to physical mock-up. a Digital waxup using the AR smile projection. b 3D-printed model of the digital wax-up. c Validation using a physical mock-up
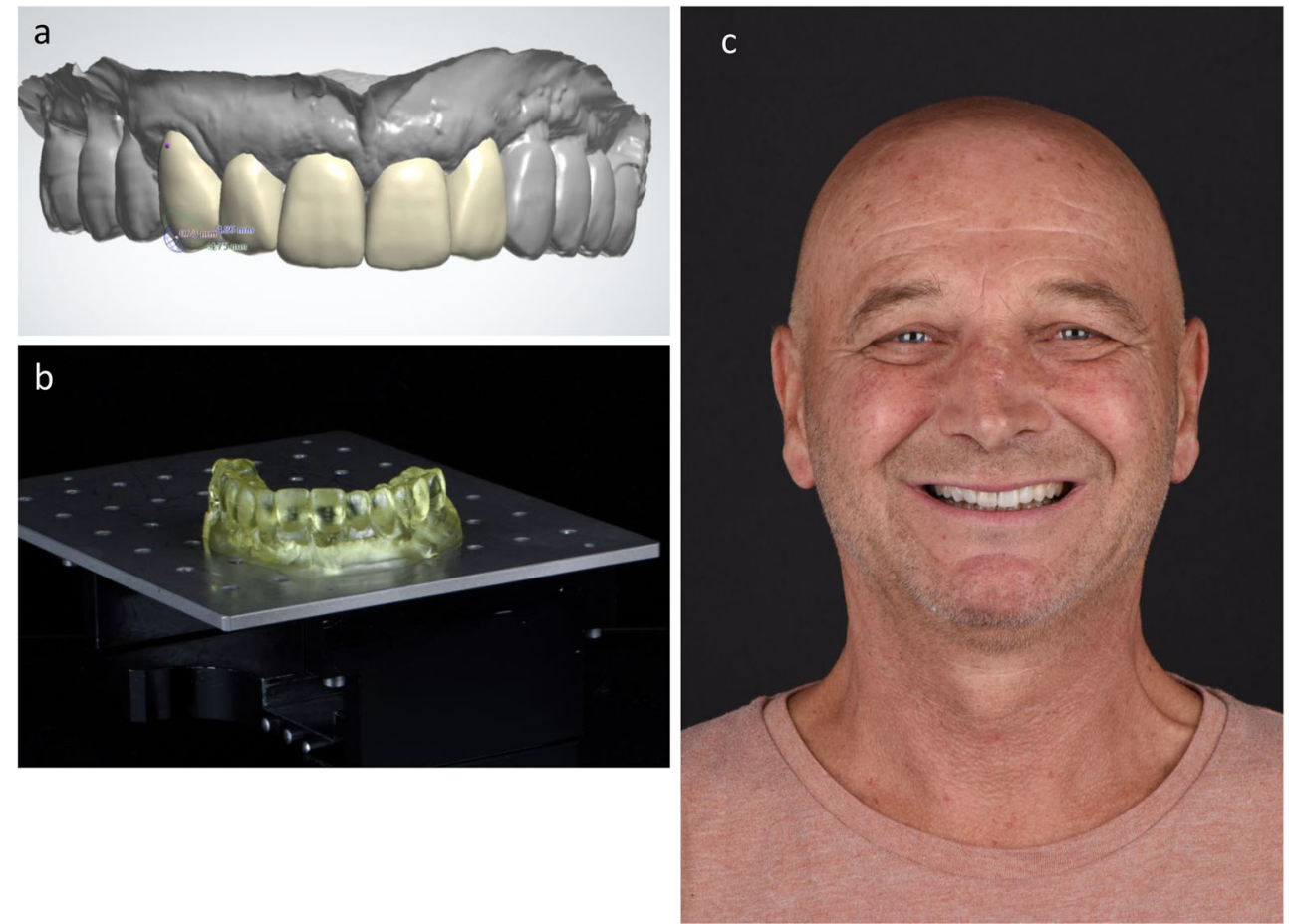

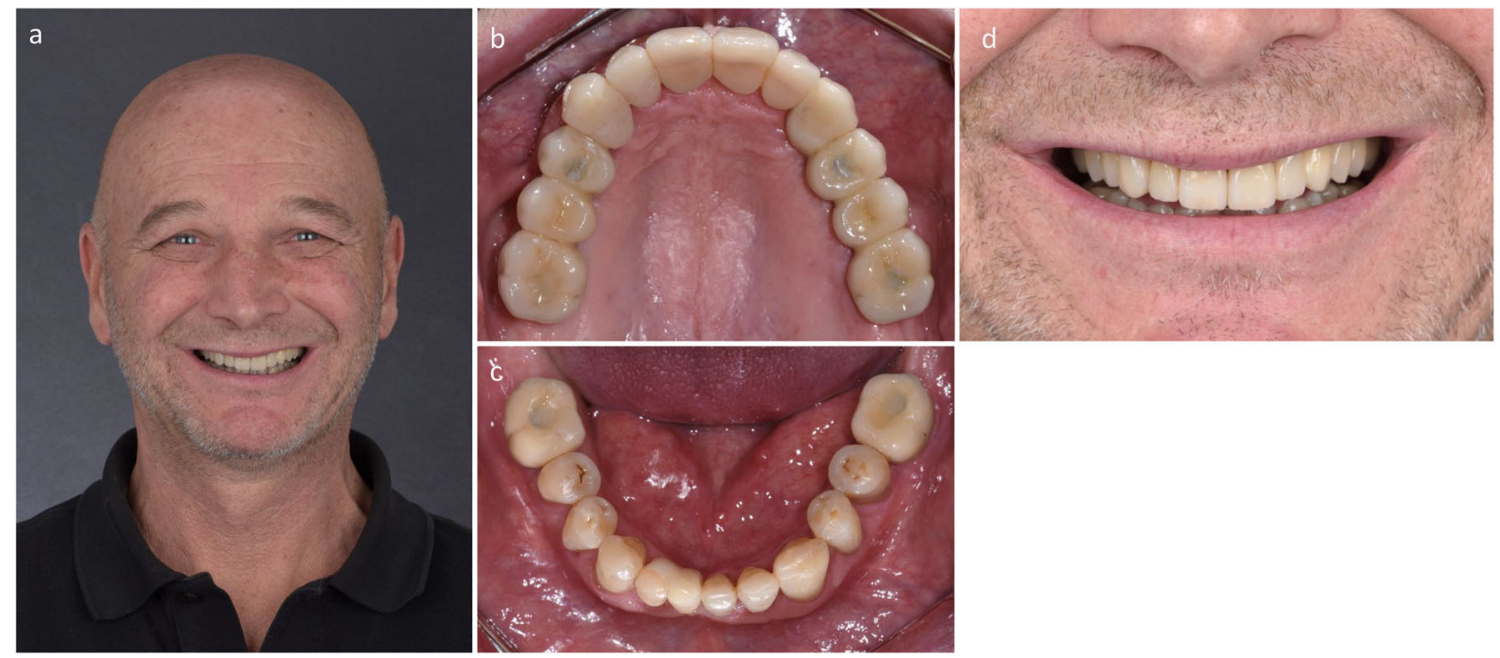

Fig. 6 Final situation. a Frontal view. b Occlusal view of the upper arch max. c Occlusal view of the lower arch. d Close up

\section{Discussion}

The present case report shows a patient with several missing teeth in both jaws as well as a compromised functional, phonetic, and esthetic situation. AR technology was used to integrate the patient into the decision-making process for reconstruction of the anterior esthetic zone [11].

Traditional smile design protocols are very effective for communication between the interdisciplinary dental team and the dental technician $[7 \cdot, 14,15]$. In those protocols, a first session is mandatory to realize photographs as well as videos [14]. Data are then analyzed by the practitioners and the dental technician to create a new smile proposition that will be exposed to the patient on a second appointment [14, 15]. Patients are mostly passive regarding the esthetic diagnosis and design, because they can comment and address his demands only after one or two propositions are already done.

In the presented protocol using AR, the patient is enabled to be an active member of the reconstructive team, as a coauthor of his rehabilitation. The patient is given the opportunity to explore the possibilities of his smile reconstruction and gives input by using a tool that helps remove language and technical barriers. This technology proposes to overhaul the organization of the first appointment in esthetic dentistry by taking less time for photos and videos acquisition and more time for discussion with the patient. In the presented patient case, it has been decided to use the device in a second phase, after stabilizing the posterior area and validating the VDO. This was necessary to ensure a correct matching of the AR smile projection and allow for increased precision. This technology may present a paradigm shift in communication with both the patient and the dental laboratory. Indeed, thanks to a new tool called "CAD-link" [12], it enables the direct matching of the final AR proposal with the digital impression in order to create a digital wax-up. This strategy is likely more time efficient when compared to protocols proposed in recent publications that mainly required conversion of the digital smile design into a digital wax-up, with supplementary manual steps executed by the technician $\left[8,160^{\circ}\right]$.

The AR tool used in this case report still presents several limitations, such as the increased knowledge required in the handling of digital technologies. The AR tool also represents a certain learning curve for patient and practitioner. The optimal use of this device depends on the accuracy when matching the 3D data acquired by the intraoral scanning system and the AR smile proposal. This step is highly sensitive as the camera of the tablet device only offers a single frontal view [11, 17]. Indeed, the matching technology uses algorithms that require coincident areas positioned under different planes [18]. In the presented patient case, the initial situation offered insufficient compatible fixpoints for a correct AR smile projection. Therefore, in situations where multiple teeth are missing, caution should be taken when applying AR technology. The lack of landmarks may create an imprecision in the smile projection of unknown degree. Finally, further technological evolutions of AR may open the spectrum of its application in dentistry, but more research in this field is necessary.

\section{Conclusion}

Co-diagnostic use of AR software may enhance the communication strategy between the clinician, dental technician, and the patient. In the case of full mouth rehabilitation, the use of AR software is recommended after stabilizing the posterior area. Further studies are needed to assess the precision and reproducibility of the described software and protocol.

Funding Open Access funding provided by Université de Genève. 


\section{Compliance With Ethical Standards}

Conflict of Interest The authors declare no competing interests.

Open Access This article is licensed under a Creative Commons Attribution 4.0 International License, which permits use, sharing, adaptation, distribution and reproduction in any medium or format, as long as you give appropriate credit to the original author(s) and the source, provide a link to the Creative Commons licence, and indicate if changes were made. The images or other third party material in this article are included in the article's Creative Commons licence, unless indicated otherwise in a credit line to the material. If material is not included in the article's Creative Commons licence and your intended use is not permitted by statutory regulation or exceeds the permitted use, you will need to obtain permission directly from the copyright holder. To view a copy of this licence, visit http://creativecommons.org/licenses/by/4.0/.

\section{References}

Papers of particular interest, published recently, have been highlighted as:

- Of importance

1. Carmigniani J, Furht B, Anisetti M, Ceravolo P, Damiani E, Ivkovic M. Augmented reality technologies, systems and applications. Multimed Tools Appl. 2011;51(1):341-77.

2. Caudell TP, Mizell DW. Augmented reality: an application of heads-up display technology to manual manufacturing processes. In: Proceedings of the Twenty-Fifth Hawaii International Conference on System Sciences, vol.2. Kauai: IEEE; 1992, p. 659-69. http://ieeexplore.iee.org/document/183317/

3. Louis Rosenberg. The use of virtual fixtures as perceptual overlays to enhance operator performance in remote environments. 1992.

4. Huang T-K, Yang C-H, Hsieh Y-H, Wang J-C, Hung C-C. Augmented reality (AR) and virtual reality (VR) applied in dentistry. Kaohsiung J Med Sci. 2018;34(4):243-8. Interesting input on new technologies used in dentistry.

5. Kwon H-B, Park Y-S, Han J-S. Augmented reality in dentistry: a current perspective. Acta Odontol Scand. 2018;76(7):497-503.

6. Vávra $\mathrm{P}$, Roman J, Zonča $\mathrm{P}$, Ihnát $\mathrm{P}$, Němec $\mathrm{M}$, Kumar J, et al. Recent development of augmented reality in surgery: a review. J Healthc Eng. 2017;2017:1-9.

7. Coachman C, Georg R, Bohner L, Rigo LC, Sesma N. Chairside 3D digital design and trial restoration workflow. J Prosthet Dent. 2020;
https://linkinghub.elsevier.com/retrieve/pii/S002239131930695X; 124:514-20. Presentation of a chairside app for digital smile design that is not using AR.

8. Stanley M, Paz AG, Miguel I, Coachman C. Fully digital workflow, integrating dental scan, smile design and CAD-CAM: case report. BMC Oral Health. 2018;18(1). https://bmcoralhealth. biomedcentral.com/articles/10.1186/s12903-018-0597-0

9. Galibourg A, Brenes C. Virtual smile design tip: from 2D to 3D design with free software. J Prosthet Dent. 2019;121(5):863-4.

10. Lavorgna L, Cervino G, Fiorillo L, Di Leo G, Troiano G, Ortensi $\mathrm{M}$, et al. Reliability of a virtual prosthodontic project realized through a 2D and 3D photographic acquisition: an experimental study on the accuracy of different digital systems. Int J Environ Res Public Health. 2019;16(24):5139.

11. Touati R, Richert R, Millet C, Farges J-C, Sailer I, Ducret M. Comparison of two innovative strategies using augmented reality for communication in aesthetic dentistry: a pilot study. J Healthc Eng. 2019;2019:1-6.

12. Marchand L, Touati R, Fehmer V, Ducret M, Sailer I. Latest advances in augmented reality technology and its integration into the digital workflow Neueste Entwicklungen in der AugmentedReality-Technologie und ihre Integration in den digitalen Workflow. Int J Comput Dent. Accepted.

13. Scaminaci Russo D, Cinelli F, Sarti C, Giachetti L. Adhesion to zirconia: a systematic review of current conditioning methods and bonding materials. Dent J. 2019;7(3):74.

14. Coachman C, Calamita M. Digital smile design: a tool for treatment planning and communication in esthetic dentistry. Quint Dent Tech. 2012;2012:1-9.

15. Coachman C, Calamita MA, Sesma N. Dynamic documentation of the smile and the $2 \mathrm{D} / 3 \mathrm{D}$ digital smile design process. Restorative Dent. 2017;37(2):12.

16. Lin W-S, Harris BT, Phasuk K, Llop DR, Morton D. Integrating a facial scan, virtual smile design, and 3D virtual patient for treatment with CAD-CAM ceramic veneers: a clinical report. J Prosthet Dent. 2018;119(2):200-5. Description of a new technology for smile design using a facial scan but not chairside.

17. Zimmermann M. Virtual smile design systems: a current review Virtuelle Smile Design-Systeme: eine aktuelle Übersicht. Int J Comput Dent. 2015;18(4):303-17.

18. Richert R, Goujat A, Venet L, Viguie G, Viennot S, Robinson P, et al. Intraoral Scanner Technologies: a Review to Make a Successful Impression. J Healthc Eng. 2017;2017:1-9.

Publisher's Note Springer Nature remains neutral with regard to jurisdictional claims in published maps and institutional affiliations. 